\title{
Is the magnetic field in quiescent prominences force-free?
}

\author{
U. Anzer ${ }^{1}$ and P. Heinzel ${ }^{2,1}$ \\ 1 Max-Planck-Institut für Astrophysik, Karl-Schwarzschild-Str. 1, 85740 Garching, Germany \\ e-mail: ula@mpa-garching.mpg.de \\ 2 Astronomical Institute, Academy of Sciences of the Czech Republic, 25165 Ondřejov, Czech Republic
}

Received 27 November 2006 / Accepted 23 February 2007

\begin{abstract}
Aims. We describe under which conditions the magnetic fields of quiescent prominences are force-free and under which gravity plays the dominant role.

Methods. The existing observational determinations of the magnetic field are summarised and the calculation of the plasma $\beta$ is outlined. We derive the dependence of $\beta$ on the prominence weight and the field strength.

Results. We show that in many cases of well-developed quiescent prominences the field can deviate substantially from the force-free situation and gravity fully determines the structure of the magnetic dips.
\end{abstract}

Key words. Sun: prominences - Sun: magnetic fields - Sun: filaments

\section{Introduction}

Solar prominences have been studied in great detail for many years. An excellent summary on that subject is the book by Tandberg-Hanssen (1995). The prominences can be divided into two basic categories: quiescent prominences and active region prominences. The former ones occur preferentially at high solar lattitudes, in particular the so-called polar crown filaments. They are in general very stable and also have large spatial dimensions. The second type is associated with active regions, they are extremely dynamic and are generally of much smaller size. Also the magnetic fields related to them differ in strength and orientation for the two classes. Therefore it is important to distinguish clearly between these two different categories when one develops models for their equilibria. In this paper we concentrate on the case of quiescent prominences.

It is widely accepted that the support of the dense and cool prominence material is due to magnetic forces. A detailed discussion of different types of magnetic support can be found in Anzer (1989). These fields must contain dips in order to lead to stable configurations. In our analysis we neglect the presence of prominence fine structure, which could complicate the modelling. These fine structures were recently reviewed by Heinzel (2007).

The mechanisms which can produce the required dips can either lie in the complex topology of the fields if the plasma beta is very low, or result from the weight of the cool prominence plasma. The crucial parameter which distinguishes between these two possibilities is the value of $\beta$ in the interior of the prominence, where $\beta=8 \pi p / B^{2}$. This implies that one needs to know both the field strength, $B$, and the gas pressure, $p$, within the prominence. For this reason we shall discuss in Sect. 2 the determinations of $B$, both by direct and indirect methods. The determination of the gas pressure inside the prominence is presented in Sect. 3. In Sect. 4 we briefly summarise the models for magnetic dips based upon force-free field calculations. In Sect. 5 we study magnetic equilibria where gravity is important. For these models a simple relation between the value of $\beta$ and the column mass has been found, which allows an independent determination of $\beta$. Section 6 summarises the conclusions.

\section{Values of the magnetic field strength}

We summarise the values of $B$ that can be found in the literature. The most direct way to determine the field is to look at prominences on the limb. These field measurements are mainly by Leroy and his coworkers. Bommier et al. $(1986,1994)$ studied a set of 14 prominences and found $B=2-20 \mathrm{G}$. An inspection of their Table 1 shows that there is a slight trend for weaker fields to occur at larger lattitudes. For lattitudes with $\Phi>35^{\circ}$ one obtains a mean value of $\bar{B}=8 \mathrm{G}$ and for $\Phi<35^{\circ}$ a mean value of $\bar{B}=10 \mathrm{G}$. We suggest that the high lattitude prominences are the best candidates to represent the quiescent prominences. Leroy et al. (1983) have also collected data on a very large number of polar crown filaments. They found $B=2-15 \mathrm{G}$ and an increase from $\bar{B}=6 \mathrm{G}$ to $\bar{B}=12 \mathrm{G}$ in the course of the solar cycle. Athay et al. (1983) obtained for a sample of 13 cases fields between 6 and $27 \mathrm{G}$. They did not distinguish between quiescent and eruptive prominences. This could be the reason why they found more cases with larger $B$.

In some more recent studies by López Ariste \& Casini (2003) and Casini et al. (2003) much stronger fields were reported. But these authors selected a small number of cases for which it is not clear that all these prominences are actually of the quiescent type. Moreover, all these new measurements were obtained without coronographs and therefore are restricted to low heights above the the limb where the prominences are bright enough and where the fields are rather strong (López Ariste 2006). For these reasons it seems clear that one would need a detailed systematic study based upon a large number of quiescent prominences to really prove that the earlier values of $B$ were systematically too low. On the basis of the presently available observations we conclude that at present nothing definite can be said about such very strong prominence fields. 
A quite different approach to determine $B$ was taken by Aulanier \& Démoulin (2003). They used the observed photospheric flux distribution around some filaments seen on the disk. Then they extrapolated the fields into the corona assuming constant $\alpha$ force-free fields. Although the assumtion of a constant value of $\alpha$ may not be correct their results could at least give the right order of magnitude of the field in the prominence. They selected one example of a quiescent prominence and calculated $B=2.5-3 \mathrm{G}$. They also had one plage prominence which gave $B=34-45 \mathrm{G}$. This shows that there is a very large difference in field strength between quiescent and active region prominences and one should not mix the two. For a detailed study of field determinations see the review by López Ariste (2007).

Based upon this discussion we shall take the standpoint that in quiescent prominences the field strength typically lies between 3 and $15 \mathrm{G}$, with a slight preference of lower values.

\section{Determination of the gas pressure}

The electron density, $n_{\mathrm{e}}$, in prominences has been derived from the Stark effect of high Balmer lines (see the discussion in Hirayama 1985). For typical quiescent prominences values between $3 \times 10^{10} / \mathrm{cm}^{3}$ and $3 \times 10^{11} / \mathrm{cm}^{3}$ have been found (Hirayama 1985, see also the Hvar Reference Atmosphere of Quiescent Prominences, Engvold et al. 1990). For the total gas pressure one has

$p=\left(n_{\mathrm{H}}+n_{\mathrm{e}}\right) k T$,

where $n_{\mathrm{H}}$ is the total number density of hydrogen. Here the small correction due to the admixture of helium has been neglected. If we introduce the ionisation degree of hydrogen, $i=n_{\mathrm{e}} / n_{\mathrm{H}}$, we get the relation

$p=n_{\mathrm{e}}(1+1 / i) k T$.

For the case of a fully ionised plasma we get a lower limit for $p$ (if the value of $n_{\mathrm{e}}$ is given) of

$p=2 n_{\mathrm{e}} k T$.

More realistic values for $i$ are around 0.3, as in the models of Anzer \& Heinzel (1999). This leads to

$p=4.3 n_{\mathrm{e}} k T$.

For $T=8000 \mathrm{~K}$ and $n_{\mathrm{e}}$ in the range between $3 \times 10^{10} / \mathrm{cm}^{3}$ and $10^{11} / \mathrm{cm}^{3}$ one derives a gas pressure of

$p=0.05-0.5 \mathrm{dyn} / \mathrm{cm}^{2}$.

Taking $i=0.07$ as suggested by Hirayama (1985) would result in even larger gas pressures $\left(0.15\right.$ to $\left.1.5 \mathrm{dyn} / \mathrm{cm}^{2}\right)$. For a representative value of the magnetic field strength of $5 \mathrm{G}$ we obtain $\beta=0.05-0.5$ (for $i=0.3$ ). Although all these quantities contain rather large uncertainties there is strong observational evidence that the magnetic field in many quiescent prominences will not be force-free. An independent method to determine $\beta$ which supports this argument will be described in Sect. 5 .

\section{Dips in force-free fields}

If we take for the magnetic field strength a representative value of $B=5 \mathrm{G}$ we have a magnetic pressure of $p_{\mathrm{m}}=1 \mathrm{dyn} / \mathrm{cm}^{2}$. For the corona we assume $n_{\mathrm{e}}=n_{\mathrm{p}}=10^{8} / \mathrm{cm}^{3}$ and $T=10^{6} \mathrm{~K}$. This gives a coronal gas pressure $p_{0}=0.03 \mathrm{dyn} / \mathrm{cm}^{2}$ and therefore one has in this region $\beta_{0}=0.03 \ll 1$. This means that the coronal field will always be force-free. As long as the gas pressure inside the prominence is also around $p_{0}$ one obtains $\beta \approx 0.03$. Therefore all prominences which have such a low central pressure will be approximately force-free and if the field is stronger than $5 \mathrm{G}$ then even larger values for the gas pressure are allowed. Under these circumstances the magnetic dips which are required for equilibrium have to be of the force-free type. These dips can be produced basically in two different ways: either by the presence of sufficiently complex photospheric flux distributions or by strong photospheric shearing motions.

For the first category the simplest case is a quadrupolar potential field as dicussed by Anzer (1990). More realistic models were developped by Aulanier \& Démoulin (1998, 2003) and Aulanier \& Schmieder (2002). They studied periodic configurations and assumed a sufficiently large number of "parasitic" polarities. Such models could simulate to a certain degree the observed photospheric flux distribution. The fields in the corona were then taken as constant $\alpha$ force-free configurations. The assumption of a constant value for $\alpha$ is certainly somewhat questionable. The other shortcoming of this approach is that the maximum value for $\alpha$ is determined by the size of the box of the numerical domain which is an unphysical constraint. But in spite of all this the investigations give some interesting new results. The authors found that in many cases large regions with magnetic dips exist in the solar corona. One can then interprete the locations of these dips as the position of the prominence.

The second category of models is based upon the presence of photospheric shearing and an efficient field line reconnection in the corona. On this basis van Ballegooijen \& Martens (1989) generated flux rope models with extended dip regions. Their model is similar to that of Priest et al. (1989) discussed below. More recently fully 3D configurations of this type were studied numerically by by DeVore \& Antiochos (2000), Aulanier et al.(2002) and DeVore et al. (2005). All these latter calculations start with some initial, usually potential, field and then apply to it a specific shearing motion. They also allow for a certain degree of reconnection within the region. As a result one obtains force-free fields for which $\alpha$ is not constant. Under certain circumstances these fields can develop regions with dips where then prominences can form. The models calculated so far apply very specific shear profiles. They have an enormous stretching present in a very narrow region. Typically the total stretching is about 12 times the lateral distance of the maximum in the shear profile. Such flows seem unrealistic in the solar photosphere. Since at present no studies exist with more realistic field line displacements it is not clear how representative the results of these calculations are.

Instead of applying some photospheric shearing to a magnetic arcade one can also use the twisting of a bipolar region to produce a force-free flux rope with internal twist. A large flux rope of this type was proposed by Priest et al. (1989) and later this type of model was extended by Lionello et al. (2002). If the configuration is sufficiently twisted then magnetic dips will develop in the lower parts of the flux rope which can be the locations of the prominence. Therefore this mechanism could be a possible alternative. The twisted flux rope configurations could also result from the emergence of field structures from below the photosphere as advocated by Fan \& Gibson (2003, 2004). There is, however, a problem with this approach: the flux tubes below the solar surface contain plasma of a very high density. Most of this material will be trapped in the magnetic dips and is therefore lifted into the corona. Since this is not very plausible one needs 
an additional mechanism by which a sufficient amount of gas is drained from the tube during its emergence.

Models with twisted flux ropes were also discussed by Low (2001) in the context of coronal mass ejections which are associated with prominence eruptions.

\section{Dips produced by the prominence}

The other possibility is that the weight of the prominence is large enough to produce the magnetic dips, or enhance existing ones. In this case the field no longer will be force-free and the value of $\beta$ in the central parts of the prominence can be very large.

We shall study these effects using simple 1D slab configurations. The details of this type of model can be found for example in the review of Heinzel \& Anzer (2005) and we give here only those relations directly relevant to our investigation. Denoting by $B_{x}$ the $x$-component of the field, by $B_{z 1}$ the vertical field at the boundary, by $M$ the total column mass, by $p_{0}$ the gas pressure of the ambient corona and by $p_{\text {cen }}$ the gas pressure in the center of the prominence we have the relations

$M=\frac{B_{x} B_{z 1}}{2 \pi g}$

and

$p_{\text {cen }}=\frac{B_{z 1}^{2}}{8 \pi}+p_{0}$.

We also allow for some magnetic shear by introducing a field component $B_{y}$ along the prominence. The field strength in the middle of the prominence is then given by

$B=\sqrt{B_{x}^{2}+B_{y}^{2}}$.

We now write this as

$B_{x}=B \sin \gamma$,

where $90^{\circ}-\gamma$ is the angle of the shearing. For simplicity we take $p_{0} \ll p_{\text {cen }}$, which is justified in most cases and we therefore set

$p_{\text {cen }}=\frac{B_{z 1}^{2}}{8 \pi}$.

Note: the generalisation to the case of $p_{0} \neq 0$ is straightforward. We define now by $\beta$ the value of $p_{\mathrm{g}} / p_{\mathrm{m}}$ in the center of the prominence. Using Eqs. (6), (9) and (10) we obtain the final result

$\beta=\left(\frac{2 \pi g M}{\sin \gamma B^{2}}\right)^{2}$.

This equation directly relates the central value of $\beta$ to the column mass $M$ and therefore the weight of the prominence. Therefore one finds that if $M$ is sufficiently large for a given field strength then $\beta$ also can become rather large, since it increases with $M^{2}$.

As a next step we derive estimates for the column mass $M$ of a prominence seen on the limb. Such estimates can be obtained from a relation which gives the column mass as a function of the optical thickness observed in $\mathrm{H} \alpha$. For this we use the tables produced by Gouttebroze et al. (1993). They considered a large grid of isobaric isothermal 1D slab models. Selecting a subset of these models that have temperatures between $6000 \mathrm{~K}$ and $8000 \mathrm{~K}$ we obtain the relation shown in Fig. 1. If we now assume that the optical thickness in the $\mathrm{H} \alpha$ line lies in the range

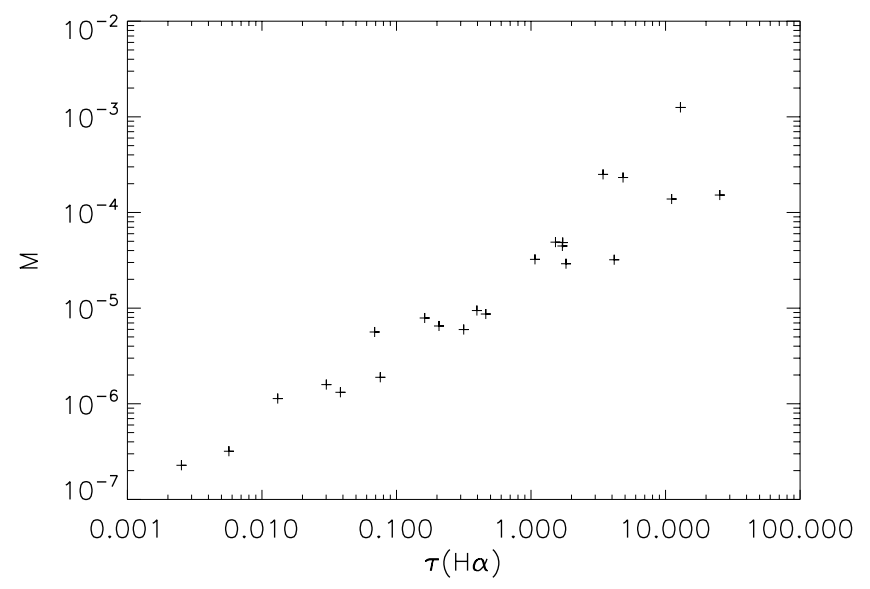

Fig. 1. Column density $M$ in $\mathrm{g} \mathrm{cm}^{-2}$ as a function of the optical thickness in $\mathrm{H} \alpha$ for a set of 1D slab models.

between 3 and 5, which seems a reasonable assumption for well developed prominences, we find the estimates

$3 \times 10^{-5} \mathrm{~g} / \mathrm{cm}^{2}<M<3 \times 10^{-4} \mathrm{~g} / \mathrm{cm}^{2}$.

Taking $B=5 \mathrm{G}$ and assuming a shear angle $\gamma$ of $30^{\circ}$ we then obtain

$0.17<\beta<17$

From our Eq. (11) one sees that $\beta$ depends on the values for $M, B$ and $\gamma$. All these values can vary over a large range. This in turn results in a wide range of expected $\beta$ values. But as the above discussion shows, values for $\beta$ between 0.3 and 10 are expecteted, if we exclude the most extreme cases. These values for $\beta$ are even larger than those obtained in Sect. 3 which strengthens our conclusion that a large number of prominences have fields which will not be force-free.

The estimates for $\beta$ can increase beyond the estimates given above when one includes the effects of prominence fine structure. In this case the value of $\tau_{\mathrm{H} \alpha}$ gives the column mass averaged along the line-of-sight whereas the actual local densities can be considerably higher. This will lead to a further increase in $\beta$.

The value of $\beta$ can also be related to the amount of field line sagging (Heinzel \& Anzer 1999). If we define the sagging angle $\phi$ by

$\tan \phi=\frac{B_{z 1}}{B}$,

we obtain the relation

$\beta=(\tan \phi)^{2}$

This equation was initially derived for 1D slab models of the Kippenhahn-Schlüter type, but it also applies to our 2D vertical thread models. In addition it will be valid approximately for all those prominence structures for which the vertical scale is significantly larger than the prominence width. This result then implies that if one can measure the full field vector at the surface of the prominence one also obtains an estimate for $\beta$. The values of $\phi$ between $30^{\circ}$ and $45^{\circ}$ found by Bommier et al. (1994) lead to $\beta$ between 0.3 and 1.0. This is independent support of our previous estimates. 


\section{Discussion}

On the basis of the presently available determinations of the magnetic field in prominences there is clear evidence that massive, well-developed prominences are not in a force-free equilibrium. We have derived estimates for the values of $\beta$ in prominences, either by using the gas pressure in the central parts of the prominence or by taking the column density together with a simple equilibrium model. We find $\beta=0.05-1.5$ from density estimates and $\beta=0.2-10$ from the column masses. Therefore values of $\beta$ between 0.2 and 1.5 are reasonable. This then implies that any prominence model will have to take this result into account by including the gravitational force which is exerted on the field. Nevertheless there can be configurations where either $B \gg 5 \mathrm{G}$, or $\tau_{\mathrm{H} \alpha}<1$, occur leading to low values of $\beta$ and therefore force-free fields will give the correct description under these particular circumstances.

All our estimates given here were derived for rather uniform distributions of the gas pressure, the column mass and the magnetic field. But the observations indicate that prominences usually show a large amount of fine structure, both as vertical threads and as horizontal fibrils. This then means that locally the values of $\beta$ can be considerably larger than our values. But these stronger deviations from force-free states will be very local. Therefore on the large scales the field configurations will be fully determined by the mean quantities discussed in this paper. An interesting aspect in this context is the fact that Aulanier \& Démoulin (2003) calculated coronal fields of 2.5-3 G for a quiescent prominence whereas typical observed fields are about a factor 2 larger. This can be explained by the compression of the initial coronal field by the weight of the prominence.

There is also the aspect that in the corona itself one has typically $p_{0} \approx 0.03 \mathrm{dyn} / \mathrm{cm}^{2}$. This implies that essentially all coronal fields are force-free. If then a prominence forms in the corona the initial field must have dips which result from the force-free configuration itself. This means that the initial shape of the prominence will be determined by the dip locations of the force-free models. But as the prominence grows in mass its weight becomes important and pulls down the field lines. This will have two effects: it enhances the existing dips and also creates new dips in the neighbourhood. From this we conclude that the fully developed prominence can occupy a much larger volume than the original dip region. But the force-free dips can be considered as seed structures for the prominence formation. The force-free models will be of some relevance but also the effects of gravity cannot be neglected.

Equilibrium models that include gravity have been developed by Anzer \& Heinzel (1998) for 1D slabs and by Heinzel \& Anzer (2001) and by Low \& Petrie (2005) for 2D threads. Similar 2D thread models were used by Petrie \& Low (2005). But in contrast to our approach they were mainly interested in the question of efficient field line reconnection and the prominence dynamics resulting from this effect. On the basis of their 2D thread configurations Heinzel \& Anzer (2006) constructed approximate 3D models for horizontal fibrils. They also solved the $3 \mathrm{D}$ radiative transfer problem in a simplified $2+1 \mathrm{D}$ approximation. This then allowed them to determine the contrast in the $\mathrm{H} \alpha$ line for different structures. For some reasonable combinations of physical parameters this $\mathrm{H} \alpha$ contrast was found to be in good agreement with the available high resolution observations of quiescent filaments on the disk.

A large number of force-free models was also used to predict the appearance of filaments on the disk (Aulanier \& Démoulin 1998; Aulanier et al. 2000, 2002; and Lionello et al. 2002).
All these investigations have in common that the structures are sketched only in a schematic way. These authors fill all the dips (or only a certain fraction of them in order to reproduce the fine fibril-like structures) up to a certain height with cool material. This height is usually taken as the pressure scale $H$ which for prominences is around $200 \mathrm{~km}$. Therefore all dips are only filled to a very small height of several hundered kilometers. Then these stuctures were taken to represent the filaments. But up to now no radiative transfer calculations were performed for these types of models. Therefore it is not clear whether the models can give the right contrast in $\mathrm{H} \alpha$ for filaments that are observed on the disk. If it turns out that the contrast is actually too low then such model filaments would be invisible. One could resolve this problem by adding more cool material and thus fill all the dips to a height of several $H$. But then the question arises whether these new configurations are still force-free or the effects of gravity become sufficiently important to require a modification of the initial models. These aspects have not been discussed so far in the literature but they should be taken into account in future modelling.

Acknowledgements. Part of this work was supported by the grant No. A3003203 of the Grant Agency of the Academy of Sciences of the Czech Republic and by the ESA-PECS project No. 98030 . We also thank the referee, G. Petrie, for his valuable comments.

\section{References}

Anzer, U. 1989, in Dynamics and Structure of Quiescent Solar Prominences, ed.

E. R. Priest (Dordrecht: Kluwer), 143

Anzer, U. 1990, SP, 130, 403

Anzer, U., \& Heinzel, P. 1998, SP, 179, 75

Anzer, U., \& Heinzel, P. 1999, A\&A, 349, 974

Athay, R. G., Querfeld, C., Smartt, R., Landi Degl' Innocenti, E., \& Bommier, V. 1983, SP, 89, 3

Aulanier, G., \& Démoulin, P. 1998, A\&A, 329, 1125

Aulanier, G., \& Démoulin, P. 2003, A\&A, 402, 769

Aulanier, G., \& Schmieder, B. 2002, A\&A, 386, 1106

Aulanier, G., DeVore, C. R., \& Antiochos, S. K. 2002, ApJ, 567, L97

Aulanier, G., Srivastava, N., \& Martin, S. F. 2000, ApJ, 543, 447

Bommier, V., Leroy, J. L., \& Sahal-Bréchot, S. 1986, A\&A, 156, 79

Bommier, V., Landi Degl'Innocenti, E., Leroy, J.-L., \& Sahal-Bréchot, S. 1994, SP, 154,231

Casini, R., López Ariste, A., Tomczyk, S., \& Lites, B. W. 2003, ApJ, 598, L67

Engvold, O., Hirayama, T., Leroy, J. L., Priest, E. R., \& Tandberg-Hanssen,

E. 1990, in Dynamics of Quiescent Prominences, ed. V. Ruždjak, \& E.

Tandberg-Hanssen, Lect. Notes Phys. (Springer), 363, 294

DeVore, C. R., \& Antiochos, S. K. 2000, ApJ, 539, 954

DeVore, C. R., Antiochos, S. K. \& Aulanier, G. 2005, ApJ, 629, 1122

Fan, Y., \& Gibson, S. E. 2003, ApJ, 589, L105

Fan, Y., \& Gibson, S. E. 2004, ApJ, 609, 1123

Gouttebroze, P., Heinzel, P., \& Vial, J.-C. 1993, A\&AS, 99, 513

Heinzel, P. 2007, in Coimbra Solar Physics Meeting on the Physics of Chromospheric Plasmas, ed. P. Heinzel, I. Dorotovič, \& R. J. Rutten, ASP Conf. Ser.

Heinzel, P., \& Anzer, U. 1999, SP, 184, 103

Heinzel, P., \& Anzer, U. 2001, A\&A, 375, 1082

Heinzel, P., \& Anzer, U. 2005, in Solar Magnetic Phenomena, ed. A. Hanslmeier, A. Veronig, \& M. Messerotti, ASSL (Springer), 320, 115

Heinzel, P., \& Anzer, U. 2006, ApJ, 643, L65

Hirayama, T. 1985, SP, 100, 415

Leroy, J. L., Sahal-Bréchot, S., \& Bommier, V. 1983, SP, 83, 135

Lionello, R., Mikić, Z., Linker, J. A., \& Amari, T. 2002, ApJ, 581, 718

López Ariste, A. 2006, private communication

López Ariste, A. 2007, in Coimbra Solar Physics Meeting on the Physics of Chromospheric Plasmas, ed. P. Heinzel, I. Dorotovič, \& R. J. Rutten, ASP Conf. Ser.

López Ariste, A., \& Casini, R. 2003, ApJ, 582, L51

Low, B. C. 2001, JGR, 106, 25141

Low, B. C., \& Petrie, G. J. D. 2005, ApJ, 626, 551

Petrie, G. J. D., \& Low, B. C. 2005, ApJS, 159, 288

Priest, E. R., Hood, A. W., \& Anzer, U. 1989, ApJ, 344, 1010

Tandberg-Hanssen, E. 1995, The Nature of Solar Prominences (Dordrecht: Kluwer Acad. Publ.)

van Ballegooijen, A. A., \& Martens, P. C. H. 1989, ApJ, 343, 971 